\title{
Seasonal infectious disease epidemiology
}

\author{
Nicholas C. Grassly* and Christophe Fraser
}

\author{
Department of Infectious Disease Epidemiology, Imperial College London, Norfolk Place, London W2 1PG, UK
}

Seasonal change in the incidence of infectious diseases is a common phenomenon in both temperate and tropical climates. However, the mechanisms responsible for seasonal disease incidence, and the epidemiological consequences of seasonality, are poorly understood with rare exception. Standard epidemiological theory and concepts such as the basic reproductive number $R_{0}$ no longer apply, and the implications for interventions that themselves may be periodic, such as pulse vaccination, have not been formally examined. This paper examines the causes and consequences of seasonality, and in so doing derives several new results concerning vaccination strategy and the interpretation of disease outbreak data. It begins with a brief review of published scientific studies in support of different causes of seasonality in infectious diseases of humans, identifying four principal mechanisms and their association with different routes of transmission. It then describes the consequences of seasonality for $R_{0}$, disease outbreaks, endemic dynamics and persistence. Finally, a mathematical analysis of routine and pulse vaccination programmes for seasonal infections is presented. The synthesis of seasonal infectious disease epidemiology attempted by this paper highlights the need for further empirical and theoretical work.

Keywords: seasons; communicable diseases; epidemiology; disease outbreaks; vaccination

\section{INTRODUCTION}

Seasonal infections of humans range from childhood diseases, such as measles, diphtheria and chickenpox, to faecal-oral infections, such as cholera and rotavirus, vector-borne diseases including malaria and even sexually transmitted gonorrhoea (Hethcote \& Yorke 1984). Despite the near ubiquity of this phenomenon, the causes and consequences of seasonal patterns of incidence are poorly understood.

This paper examines these causes and consequences, providing an overview of seasonal infectious disease epidemiology. It is divided into three sections. In the first section, the causes of seasonal patterns of human infectious disease incidence and their association with different modes of transmission are briefly reviewed. The second section provides an overview of seasonal infectious disease epidemiology, examining the consequences of seasonality for threshold parameters, such as $R_{0}$, disease outbreaks, endemic dynamics and persistence. The third section deals with the implications of seasonality for disease control by routine and pulse vaccination programmes. In the second and third sections, several new results are obtained concerning the expected size distribution of disease outbreaks and pulse vaccination strategies for seasonal infections. These results are discussed and areas for further empirical and theoretical work identified.

\section{CAUSES OF SEASONALITY}

The causes of seasonal and longer period cycles in the incidence of infectious diseases have long puzzled epidemiologists (e.g. Ransome 1880). Careful mathematical analysis in the early twentieth century led to the description of the 'law' of mass action, which states that

*Author for correspondence (n.grassly@imperial.ac.uk). the number of new infections depends on the product of the number of infected individuals, the number susceptible and a transmission parameter (called variously a 'contact rate', 'coefficient of infectivity', etc.; for a history, see Heesterbeek 2005). If infection results in protective immunity or death, the law of mass action predicts cycles in disease incidence. Soper (1929), however, made it clear that, at least in the case of measles, significant seasonal variation in the transmission parameter must also occur. One proposed cause of this seasonal variation was the congregation of children during school terms. This was confirmed about 50 years later by detailed analysis of weekly measles case reports from England and Wales, which demonstrated a decline in the transmission parameter during school holidays (Fine \& Clarkson 1982).

Although significant progress has been made towards understanding the basis of measles seasonality in temperate regions, the causes of seasonal patterns of incidence for most other infections remain unclear. We reviewed the scientific literature and citations therein using the search term 'season", AND ('infect", OR 'disease") in ISI Web of Science, PubMed and Google Scholar. This revealed a limited evidence base supporting the role of several different mechanisms in driving seasonal transmission of human infectious diseases. These can be grouped under four main headings. A similar exercise was recently conducted by Altizer et al. (2006), where infectious diseases of wildlife are also considered and the importance of seasonal reproduction of the host discussed.

\section{(a) Survival of pathogen outside host}

The survival of the pathogenic organism outside a host depends on the characteristics of the environment, particularly temperature, humidity, exposure to sunlight, $\mathrm{pH}$ and salinity. Annual variation in climate can therefore result in annual or more complex peaks in disease incidence, depending on the influence of climatic 
variables, such as rainfall or cloud cover on the environment. This relationship depends on the type of environment (e.g. sewage, aerosol, droplets, etc.), and hence route of transmission.

The most studied examples of environmentally driven seasonality are for pathogens whose predominant route of transmission is faecal-oral. The enhanced survival of rotavirus and norovirus at low temperatures is responsible for the typically observed winter peaks in gastroenteritis (Cook et al. 1990; Mounts et al. 2000). Similarly, seasonal increases in cholera incidence occur when the microenvironment favours growth of the bacterium (Pascual et al. 2000, 2002). There is, however, a complex relationship between the micro-environment and bacterial growth, which depends on temperature, salinity and overall levels of water in the environment. The incidence of cholera is therefore difficult to predict from data on climate, and may peak once or twice a year depending on the local environment (Pascual et al. 2002).

The ability to survive outside a host is also likely to be an important cause of seasonal incidence for infections transmitted by aerosols, droplets and fomites. For example, low humidity increases the survival of the influenza virus in air, and annual variation in indoor humidity has been proposed as a cause of the winter seasonality of influenza (Loosli et al. 1943; Hemmes et al. 1960). In the tropics, seasonal peaks in the incidence of respiratory syncytial virus have also been found to correlate with low humidity (Chew et al. 1998).

\section{(b) Host behaviour}

The congregation of children during school terms has been clearly demonstrated to influence annual variation in the weekly incidence of measles in England and Wales (Fine \& Clarkson 1982; Finkenstadt \& Grenfell 2000). Other studies, based on monthly data collected in Europe and the United States, report a reduction in the estimated transmission parameter over the summer months for measles and other childhood infections, including mumps, chickenpox, diphtheria and pertussis (London \& Yorke 1973; Gomes et al. 1999; Deguen et al. 2000). While this is likely to reflect the long school holiday in the summer, an explanation based solely on climate cannot be excluded because of the lack of resolution in the time-scale for reported cases.

Perhaps because of the seminal work on measles, changes in host behaviour, particularly patterns of movement and contact, have a strong tradition as explanations for the seasonality of infectious diseases. However, with the exception of childhood infections, the evidence base for this tradition is largely anecdotal. An increased rate of partner change during the summer vacation is likely to explain the peaks in gonorrhoea incidence during August to October in the United States (Hethcote \& Yorke 1984). However, there appear to be no clear examples of annual variation in contact patterns that explain, in isolation, the seasonal incidence of other infectious diseases.

\section{(c) Host immune function}

Seasonal change in host immune competence, and hence susceptibility to infection or to symptomatic disease following an infection, has been proposed as a cause of seasonal variation in the incidence of some infectious diseases of humans (Dowell 2001). Several potential mechanisms for annual variation in immune competence have been suggested, including photoperiod effects and physiological stress. However, although examples exist for infectious diseases of wildlife, evidence for the importance of these factors in the seasonal incidence of human infectious diseases is currently lacking (Nelson \& Demas 1996; Altizer et al. 2006). Perhaps the best-supported role for seasonal changes in human immune competence is the decline in mucosal integrity during the dry season in Africa and the associated increase in cases of bacterial meningitis (Greenwood 1999; Sultan et al. 2005). This is likely to be due to an increased risk of invasive disease among infected individuals, rather than increased transmission, since bacterial transmission continues during the rainy season (Blakebrough 1979).

\section{(d) Abundance of vectors and non-human hosts}

Seasonal changes in vector abundance, including mosquitoes, ticks, snails, fleas and flies, are well-documented causes of seasonality for vector-borne infections (Lord et al. 1996; Chavasse et al. 1999; Emerson et al. 2000; Sturrock et al. 2001; Randolph 2004; Mabaso et al. 2005). For example, seasonal variation in mosquito abundance in response to annual variation in temperature and rainfall can cause strongly seasonal patterns of disease incidence in malaria epidemic regions, such as the Kenyan highlands (Hay et al. 2003). Similarly, seasonal peaks in the incidence of diarrhoeal disease in Pakistan correlate with a high density of houseflies, and may be suppressed by effective fly control (Chavasse et al. 1999). The incidence of zoonoses may also be influenced by the abundance of the primary (non-human) host. For example, plague dynamics in central Asia are influenced by the abundance of their primary gerbil host (Davis et al. 2004).

\section{THE EPIDEMIOLOGY OF SEASONAL INFECTIONS}

\section{(a) The basic reproductive number}

The basic reproductive number $R_{0}$ for a non-seasonal infection is typically defined as the number of secondary infections that result from the introduction of a single infectious individual into an entirely susceptible population (Anderson \& May 1991). This interpretation is not possible for seasonal infections, since the number of secondary infections will depend on the time of year that the infectious individual is introduced.

In the non-seasonal case, $R_{0}$ is a threshold parameter, with $R_{0}>1$ implying initially exponential growth in the number of infections. It may be estimated by analysis of the stability of the system of ordinary differential equations describing transmission, linearized about the infectionfree equilibrium (e.g. Diekmann et al. 1990). Linearization means the impact of the epidemic on the number of susceptible individuals is ignored. A similar approach can be used to define threshold parameters for seasonal infections, and involves linearization over the entire year. The conditions for stability of the infection-free equilibrium can then be established using Floquet's theorem (Heesterbeek \& Roberts 1995). However, this approach often requires numerical solution of the linearized system over one year. Furthermore, to derive a threshold parameter that has a similar interpretation to $R_{0}$ requires averaging over all possible times of year that an infection 
may be introduced. It would therefore be helpful if suitable approximations for threshold parameters analogous to $R_{0}$ could be derived in the seasonal case.

Work in this direction suggests that for a general class of functions describing the distribution of the infectious period, including exponential, the arithmetic mean of the transmission parameter scaled by the duration of infection gives the appropriate threshold parameter to a very good approximation (Williams \& Dye 1997). We therefore define a basic reproductive number for a seasonal, homogeneous susceptible-infected-recovered (SIR) model by

$\bar{R}_{0}=D \int_{0}^{1} \beta(t) \mathrm{d} t$,

where $\beta(t)$ is the transmission parameter at time $t$ years and $D$ the average duration of infection. This may be interpreted as the average number of secondary cases arising from the introduction of a single infected person into a completely susceptible population at a random time of the year. The condition $\bar{R}_{0}<1$ is not sufficient to prevent a (potentially large) outbreak, since chains of transmission can be established during the high season if $D \beta(t)>1$, but is sufficient and necessary for long-term disease extinction. To prove this mathematically, it is sufficient to note that extinction must be possible in the linearized model where the fraction of the population susceptible is assumed to be 1. In this case, change in the fraction infected $y$ is given by $D \mathrm{~d} y / \mathrm{d} t=(D \beta(t)-1) y$, the solution of which shows $y \rightarrow 0$ as $t \rightarrow 0$ if and only if $\bar{R}_{0}<1$. This proof can be extended for certain types of population heterogeneity, where the amplitude of seasonal forcing is independent of who infects who, but is surprisingly complex as soon as additional infection stages are introduced (as is the case for the susceptible-exposed-infected-recovered (SEIR) model; see Williams \& Dye 1997; Ma \& Ma 2006). In case of the latter, equation (3.1) then only defines a numerically approximate basic reproductive number.

For mathematical convenience, seasonal transmission is often assumed to be sinusoidal, such that

$\beta(t)=\beta(1+\sigma \cos (2 \pi t))$,

where $\sigma$ is the amplitude of seasonal variation in transmission (typically referred to as the 'strength of seasonal forcing'). In this case, $\bar{R}_{0}=D \beta$ as for nonseasonal infections (where $\sigma=0$ ).

Of course, sinusoidal forcing of transmission is a crude assumption for many infectious diseases, where the appropriate functional form will depend on the major cause(s) of seasonality. For example, the estimated transmission parameter for measles in the UK drops during the school holidays (figure 1). A mechanistic model for the seasonal transmission of measles is therefore often based on 'term-time forcing', such that

$\beta(t)=\beta(1+\sigma \operatorname{term}(t))$,

where term $(t)$ is +1 during the term and -1 during the holidays (Keeling et al. 2001). Simple SEIR models based on term-time forcing are capable of capturing many of the observed features of measles dynamics without resorting to more complex age-structuring (Earn et al. 2000). However, the step-function (3.3) differs from the annual variation in the transmission parameter estimated from the data, which peaks at the beginning of each term (Finkenstadt \& Grenfell 2000; figure 1). These peaks are

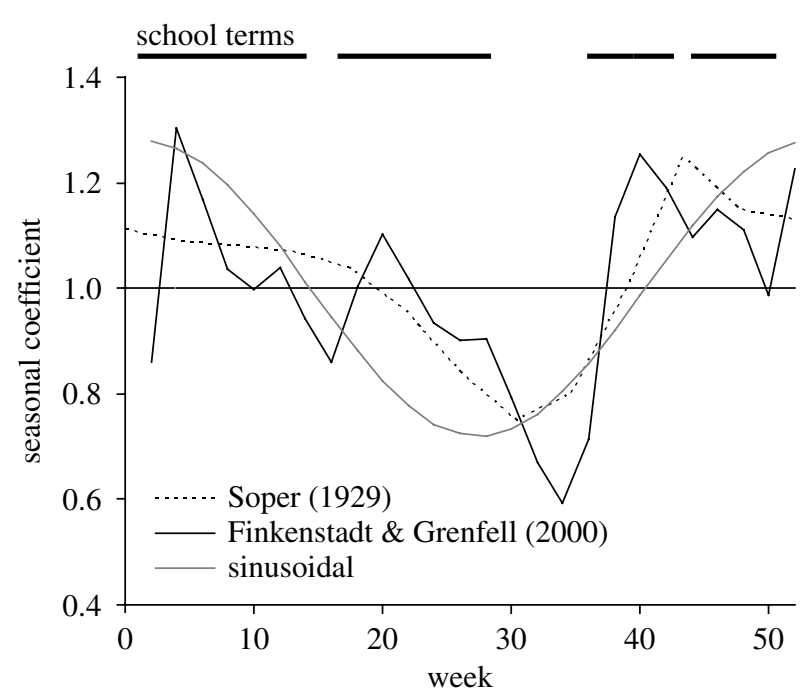

Figure 1. Estimated average seasonal transmission parameter (mean centred) for measles in the UK compared to sinusoidal forcing with $\sigma=0.28$. The monthly estimates from Soper (1929) are averages over 1905-1916 for Glasgow, and the biweekly estimates from Finkenstadt \& Grenfell (2000) are averages for England and Wales over 1944-1964. School terms are indicated at the top of the figure by the thick bars.

likely to reflect heterogeneity in transmission, with early saturation of infection among susceptible children with frequent infectious contacts.

\section{(b) Outbreaks}

In the absence of endemic disease transmission with $R_{0}<1$, outbreaks of infection may still occur following the importation of infection due to the stochastic nature of the transmission process. Surveillance systems that are able to report the duration and size of these outbreaks can provide important information about the underlying transmission parameter and contact patterns in a given population.

Ignoring the impact of the outbreak on the number of susceptible individuals, an outbreak in a simple nonseasonal SIR model of infection may be described by a branching process with a constant probability of infection $R_{0} /\left(R_{0}+1\right)$ and probability of recovery $1 /\left(R_{0}+1\right)$ (Bailey 1975; Farrington et al. 2003). The probability of observing an epidemic of size $n$ following the importation of a single case is then given by

$P(n)=\frac{(2 n-2) ! R_{0}^{n-1}}{n !(n-1) !\left(R_{0}+1\right)^{2 n-1}}$.

This elegant result has been used to estimate $R_{0}$ for measles in England and Wales and the United States from the observed distribution of outbreak sizes (Jansen et al. 2003; Gay et al. 2004). In England and Wales, this highlighted the significant increase in $R_{0}$ between 1995 and 2002 due to a decline in vaccine coverage following the combined measles-mumps-rubella (MMR) vaccine scare. Some difficulties in the application of branching process theory remain however, since population structure and associated heterogeneity in $R_{0}$ may undermine the linearity assumed in (3.4). For example, larger outbreaks of vaccine-preventable disease are frequently restricted to communities that refuse vaccination based on religious or other beliefs and in which saturation effects become important (Ramsay et al. 2003). Also, failure to link cases to particular outbreaks can lead to under-reporting of 


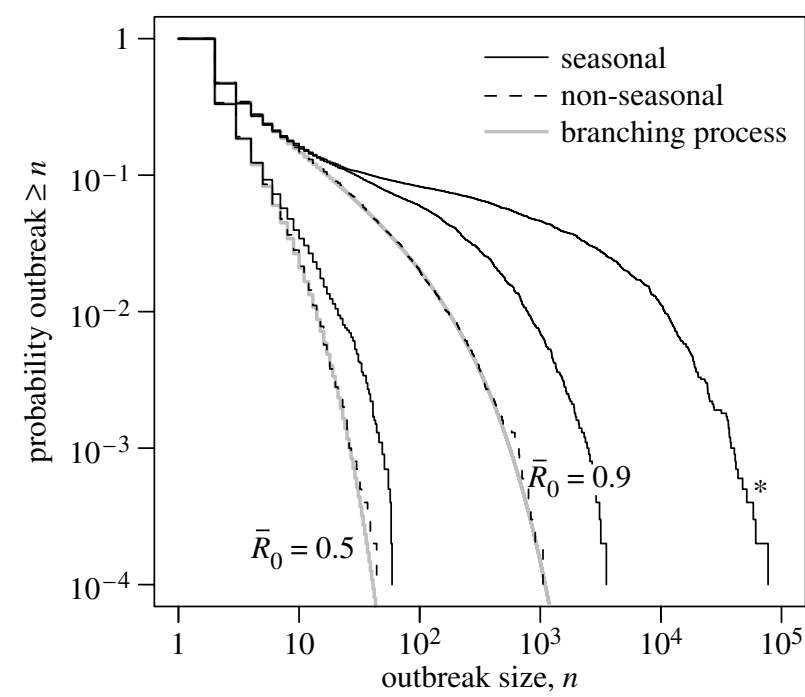

Figure 2. The distribution of final outbreak size following the introduction of a single case in a stochastic SIR model in the presence $(\sigma=0.5)$ and absence $(\sigma=0)$ of seasonal variation in the transmission parameter for different values of the average basic reproductive number $\bar{R}_{0}$. Each curve represents the outcome from 10000 single case importations occurring on a randomly distributed day of the year. The expected distribution based on branching process theory is shown in grey. We ignore the demographic processes of birth and death since outbreaks occur over a relatively short period. In this case, the probability of an infection occurring in a small time interval $\mathrm{d} t$ is $\beta(t) S(t) I(t) \mathrm{d} t / N(t)$ and that of recovery to the immune class $I(t) \mathrm{d} t / D$, where $S(t)$ and $I(t)$ are the number of susceptible and infected individuals, respectively, at time $t$, the total population size $N(t)=10^{7}$ is constant over time, $\beta(t)$ is the transmission parameter and $D=2$ weeks is the mean infectious period. The asterisked line shows the increase in larger outbreaks seen for a shorter duration of infection (one week) when $\bar{R}_{0}=0.9$ and $\sigma=0.5$.

larger outbreaks and bias estimates of $R_{0}$ downwards (Gay et al. 2004), while a tendency to link unconnected cases or preferentially investigate larger outbreaks could bias estimates upwards (Ferguson et al. 2004).

In the case of seasonal infectious diseases, including childhood infections such as measles, annual variation in the underlying transmission parameter is an important cause of temporal heterogeneity in $R_{0}$. This temporal heterogeneity alters the expected distribution of outbreaks sizes for a given annual average $\bar{R}_{0}$. The time dependence of the transition probabilities makes analytic results difficult to derive (although see Jagers \& Nerman 1985; Trapman et al. 2004), and we therefore examine the effect of seasonal variation in $R_{0}$ on the distribution of outbreak sizes numerically using a stochastic SIR model.

In the absence of seasonal variation, the distribution of outbreak sizes in the stochastic model agrees with branching process calculations (figure 2). However, seasonal variation shifts this distribution towards larger outbreaks, with the difference dependent on the amplitude and form of the seasonal variation in transmission. The effect is most marked when $D \beta(t)$ exceeds 1 during the high season, in which case epidemics can occur. These epidemics increase in size with $\bar{R}_{0}$, and for a fixed $\bar{R}_{0}$ with shorter generation times for the infection (faster recovery rate). However, even if $D \beta(t)$ never exceeds 1, autocorrelation in the transmission parameter results in larger outbreaks than predicted for non-seasonal infections. This means that estimates of $R_{0}$ from observed outbreak size distributions that ignore seasonal effects may be biased upwards. However, in the case of realistic term-time forcing of measles transmission, the extent of this bias is not large; the probability of an outbreak of more than 100 cases for $\bar{R}_{0}=0.9$ is not significantly different in the presence $(2.6 \%)$ or absence $(2.5 \%)$ of term-time forcing of transmission with $\sigma=0.25$ and term dates as described in Keeling et al. (2001).

\section{(c) Endemic dynamics}

In contrast to outbreaks, the dynamics of endemic seasonal infections have been much studied, both empirically and theoretically (Hamer 1906; Soper 1929; London \& Yorke 1973; Dietz 1976; Aron \& Schwartz 1984; Schwartz 1985, 1992; Grenfell et al. 2001; Keeling et al. 2001; Bauch \& Earn 2003; Rohani et al. 2003). The key features are briefly reviewed here to provide a background to the subsequent section on vaccination.

Oscillations in disease incidence are frequently observed, even for non-seasonal infections, where at least partially protective immunity results in a decline in the susceptible population during an epidemic. A subsequent period of low incidence follows, while the susceptible population is replenished, until an epidemic can occur again. These oscillations are predicted by stability analysis of the endemic equilibrium of deterministic models of disease transmission (e.g. Anderson \& May 1991). For example, perturbation of the endemic equilibrium of the SIR model results in damped oscillations in incidence with period $T \simeq 2 \pi \sqrt{A D}$, where $A$ is the mean age at infection at equilibrium. In the stochastic SIR model, the random nature of the transmission process continually perturbs the system and oscillations in incidence are sustained with period $T$ (figure $3 a$ ). For reasonably large population size $N$, the distribution of the number of infected individuals in this quasi-stationary state is approximately normal with variance $\sigma_{\mathrm{I}}^{2} \approx N\left(R_{0}-1\right) / R_{0}^{2}$ (Nåsell 2002).

The introduction of seasonal forcing of transmission to these models can result in complex dynamics. If $T$ is close to one year, then simple resonance results in even mild seasonal variation in transmission causing large amplitude oscillations in incidence (Dietz 1976). This may be one reason why it is difficult to identify the causes of seasonality for influenza (Dushoff et al. 2004). If $T$ is significantly larger than one year, then more complex dynamics may ensue, and stochastically driven switching occurs between different attractors of the deterministic system with different periods (figure 3b) (Schwartz 1985; Rohani et al. 2002). For example, numerical analysis of the attractors of a seasonal SIR model correctly predicts the dynamical transitions observed for the periodicity of measles epidemics in England and Wales and the United States (Earn et al. 2000). More recently, it has been shown that the stochastically driven transient behaviour of the system away from these attractors is also important, and indeed is necessary to explain the observed dynamics of rubella and whooping cough (Rohani et al. 2002; Bauch \& Earn 2003).

Increasing the strength of seasonal forcing $(\sigma)$ in simple SIR models of transmission with initially annual epidemics leads to period doubling bifurcations and eventually chaos 


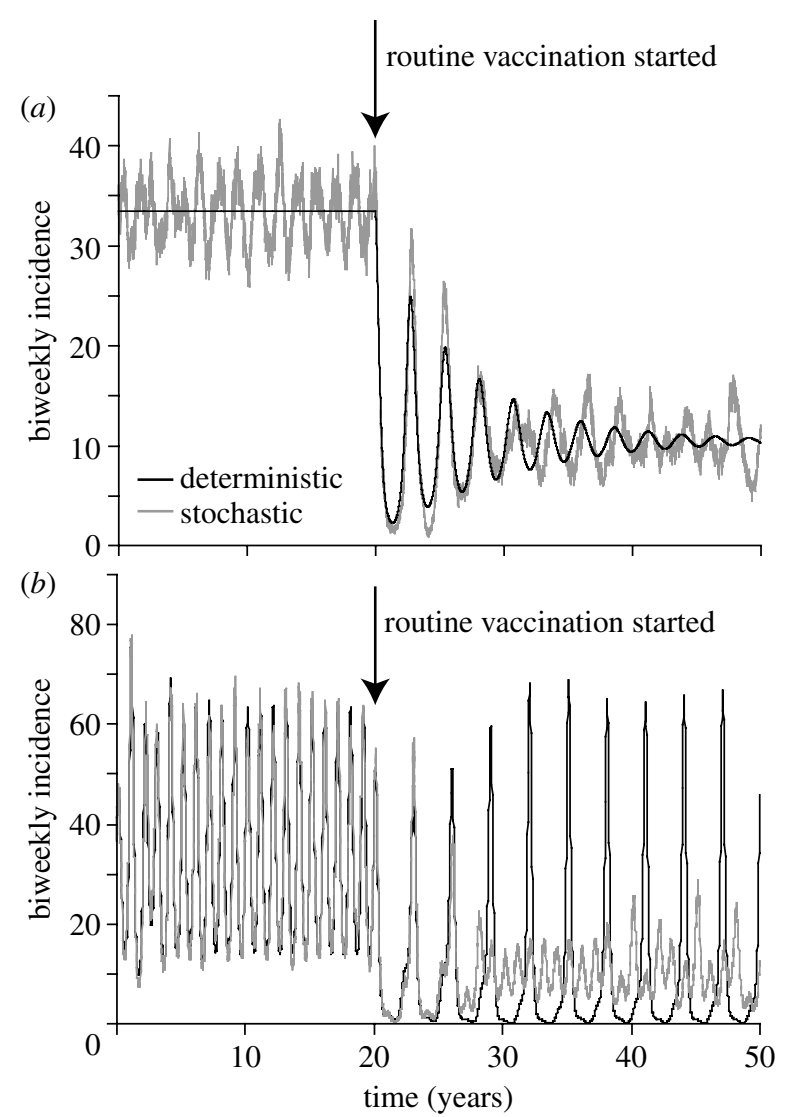

Figure 3. Endemic dynamics of deterministic and stochastic SIR models of $(a)$ non-seasonal and $(b)$ seasonal infections, with the introduction of routine vaccination of $60 \%$ of the population at birth after 20 years. (a) The non-seasonal deterministic model shows damped oscillation in incidence following perturbation of the system by vaccination, with the period $T \simeq 2 \pi \sqrt{A D}$ predicted by stability analysis of the endemic equilibrium. In the stochastic case, these oscillations are sustained both pre- and post-vaccination, although with different periods reflecting the change in the mean age at infection $A$ following vaccination. (b) The change in the natural period of the system following vaccination in the seasonal model results in a transition from annual to triennial epidemics in the deterministic case. In the stochastic model post-vaccination, more complex dynamics are observed, with switching between the attractors with annual and triennial periodicity driven by the stochasticity. These simulations are for an infection with $\bar{R}_{0}=8$, an infection period of two weeks, population size $N=10^{6}$ and rate of exit from the population $\mu=0.1$. Vaccination moves individuals from the susceptible to the recovered compartment. The seasonal model assumes a sinusoidally varying transmission parameter with $\sigma=0.1$. In the stochastic model, we assume a small rate of import of infection of $10^{-5} \mathrm{yr}^{-1} N^{-1}$ to avoid extinction of infection.

(Aron \& Schwartz 1984). In the case of measles in industrialized countries, estimates of the strength of seasonal forcing suggest chaotic dynamics driven by seasonality are unlikely (Earn et al. 2000). In fact, it now appears that the observed patterns of incidence of most seasonal childhood infections in these countries are explained by the interplay of stochasticity, nonlinear dynamics and mild seasonal forcing (Rohani et al. 2002).

\section{(d) Persistence}

Seasonal variation in transmission tends to reduce disease persistence due to the low number of cases between epidemic peaks and the possibility of stochastic extinction of infection. The importance of stochastic extinction or 'fade-out' has long been recognized, with Bartlett (1957) first drawing attention to an apparent 'critical community size' for measles, below which stochastic fade-out became important.

In the case of structured (meta-) populations, seasonal forcing of transmission tends to synchronize epidemics in different places, leading to a greater probability of stochastic extinction of infection across the entire metapopulation (Grenfell et al. 1995). However, this may not always be the case, since for certain model structures complex transient and asynchronous dynamics across the meta-population can be sustained by seasonality (Allen et al. 1993; Grenfell et al. 1995).

\section{VACCINATION AGAINST SEASONAL INFECTIONS}

\section{(a) Routine vaccination}

Routine vaccination of a fraction of the population $p$ at or soon after birth increases the mean age at infection (by $1 /(1-p)$ in a homogeneous population), and therefore increases the natural period $T$ of unforced endemic dynamics. For a seasonally forced infection, this can therefore lead to a change from regular annual or biennial epidemics to more complex patterns of disease incidence (Earn et al. 2000). In the case of measles in England and Wales, the more complex dynamics that followed vaccination led to a decline in the temporal correlation of epidemics in different cities (Bolker \& Grenfell 1996). This may have had the perverse outcome of reducing the likelihood of measles elimination.

\section{(b) Pulse vaccination}

Pulse vaccination was developed as a strategy to maximize vaccine coverage where routine programmes were failing (John et al. 1983). Disease eradication programmes using this strategy rely on the administration of large numbers of vaccine doses on 'immunization days', with the aim of maintaining the number of susceptible individuals below that sufficient to support endemic transmission. Pulse vaccination is central to the WHO strategy for the global eradication of polio (Heymann \& Aylward 2004). Theoretical work has focused on the maximum allowable inter-pulse interval, which for a homogeneous population with constant birth rate, in the absence of routine programmes, is simply $p^{\prime} A$, where $p^{\prime}$ is the fraction vaccinated during a single immunization day (Agur et al. 1993; Nokes \& Swinton 1995, 1997).

Despite most vaccine preventable infections showing seasonal variation, the theory in support of pulse vaccination has not taken this into consideration. Little thought has been given to the timing of immunization days, which typically occur during the low season with the stated aim of interrupting local transmission (Birmingham et al. 1997). In fact, the interaction of seasonal forcing of transmission with cycles in the susceptible population induced by typically annual pulse vaccination is an important determinant of the annual average reproductive number and disease dynamics.

To measure the impact of vaccination on disease transmission, it is useful to define an effective reproductive number $R_{v}$ as the number of secondary infections that results from the introduction of an infectious individual 
into a vaccinated but naive population (i.e. in the absence of density-dependent effects). In the case of a seasonal infection, the annual average $R_{v}$ is given by

$\bar{R}_{v}=D \int_{0}^{1} \beta(t) Q(t) \mathrm{d} t$,

where $Q(t)$ is the fraction of the population that is unvaccinated at time $t$.

Consider a homogeneous, annual pulse vaccination programme, such that a fraction $(1-q)$ of the susceptible population is successfully vaccinated each immunization day (and that immunity is an all-or-nothing response). At time $\tau$ after vaccination, anyone aged less than $\tau$ will be unvaccinated, a proportion $q$ of those aged between $\tau$ and $\tau+1$ will be unvaccinated, and so on all the way up to those aged between $n-1+\tau$ and $n$, a proportion $q^{n-1}$ of which will be unvaccinated. If we assume the population size does not change with age, the fraction of the population aged less than $n$ years old that remain unvaccinated at time $\tau$ after the immunization day is therefore (with some simplification) given by

$Q(\tau)=\left(1-q^{n}\right)(\tau+q /(1-q)) / n$.

In a model with sinusoidal forcing of transmission shifted by $\delta$ relative to the vaccination schedule, such that $\tau=t+\delta$, substituting (3.2) and (4.2) into (4.1) and integrating over $\tau$ gives the annual average effective reproductive number,

$\bar{R}_{v}=\beta \frac{1-q^{n}}{n}\left[\frac{1}{2}+\frac{q}{1-q}-\frac{\sigma}{2 \pi} \sin (2 \pi \delta)\right]$.

This function is minimized by vaccinating, such that $\delta=0.25$ years, in other words three months prior to the peak in the transmission parameter. The value of $\delta$ for the conventional approach of vaccinating during the season of low incidence will depend on the relative timing of peaks in incidence compared to peaks in the transmission parameter. These can differ significantly, depending on the natural history of the infection and $R_{0}$ (Dietz 1976). For many infections, peak incidence will lag peak transmission by up to about three months (e.g. Yorke et al. (1979).

The relative change in $\bar{R}_{v}$ that results from vaccinating at different times of the year depends solely on the strength of seasonal forcing $\sigma$ and the fraction vaccinated each round $q$ (figure $4 a$ ). For example, for an infection with peak transmission mid-summer and seasonal forcing $\sigma=$ 0.3 , annual vaccination of $80 \%$ of the population in April would reduce $\bar{R}_{v}$ by $6 \%$ compared to vaccinating in January (or compared to vaccinating at any time of year when $\sigma=0)$.

Similar effects can be observed for other forms of seasonal forcing. For example, in the case of term-time forcing (3.3), pulse vaccination at the end of the long summer holiday minimizes $\bar{R}_{v}$ (since the largest values of $Q(\tau)$ then occur out of school term). With $\sigma=0.3$, annual pulse vaccination of $80 \%$ of children at this time results in an $\bar{R}_{v}$ that is $28 \%$ lower than for vaccination every January. These sorts of calculation can be extended to include more realistic demography (changing population size by age), pulse vaccination with a different frequency, additional coverage through routine vaccination and different forms of seasonal forcing appropriate to the infection and location of interest.
For example, if mortality occurs at a constant rate $\mu$, that is independent of age (type-I mortality sensu Anderson \& May 1991), then we find the proportion unvaccinated is given by

$Q(\tau)=1-\mathrm{e}^{-\mu \tau} \frac{1-q}{1-q \mathrm{e}^{-\mu}}$,

and the average reproduction number is

$$
\begin{aligned}
\bar{R}_{v}=\beta & {\left[1-\frac{\left(1-\mathrm{e}^{-\mu}\right)(1-q)}{\mu\left(1-q \mathrm{e}^{-\mu}\right)}\right.} \\
& \left.\times\left(1+\sigma \frac{1}{1+\mu / 2 \pi}\left(\sin (2 \pi \delta)+\frac{\mu}{2 \pi} \cos (2 \pi \delta)\right)\right)\right] .
\end{aligned}
$$

This results in the optimal time for vaccination being brought closer to the time of peak transmission, specifically $\delta=\tan ^{-1}(2 \pi / \mu) / 2 \pi$. In practice, this correction will be small even in areas of high mortality, so that the previously derived result, $\delta \approx 0.25$, corresponding to vaccinating three months prior to peak transmission is still a reasonable approximation.

In the case that routine vaccination is also in place, if it occurs at or close to birth, then $Q(\tau)$ is simply multiplied by a constant that depends on routine vaccination coverage and efficacy, and the optimal time for pulse vaccination remains unchanged. For more complex routine immunization schedules, analytical expressions may be derived on a case-by-case basis, but these will be unwieldy and are not pursued here.

Pulse vaccination at the optimal times described above minimizes not only $\bar{R}_{v}$, but also the amplitude of the variation in $R_{v}$ over time. Such strategies can be thought of as effectively reducing the strength of seasonal forcing $\sigma$. In the absence of disease, this minimizes the probability of an outbreak and the size of any outbreaks that do occur (cf. figure 2). This makes such strategies optimal postelimination, for example in the case of polio-free countries. In the presence of disease, the decrease in $\bar{R}_{v}$ can result in a lower cumulative incidence of infection. However, persistence properties may be affected by the effective reduction in $\sigma$, since seasonal forcing typically reduces the persistence of an infection (Grenfell et al. 1995). For infections whose epidemiology and natural history result in robustly annual epidemics (e.g. polio in countries with poor sanitation), numerical simulations of a stochastic, sinusoidal seasonal SIR model suggest that any increase in persistence due to the effective reduction in $\sigma$ is more than balanced by the overall reduction in $\bar{R}_{v}$, such that both persistence and cumulative incidence are decreased by vaccinating three months prior to peak transmission (figure $4 b$ ). A similar result is found for simple meta-population versions of this model (results not shown).

Pulse vaccination can change the periodic behaviour of infections through both changes in the natural period of the system $T$ and through the introduction of an annual (or other period) forced oscillation in the number of susceptible individuals. For infections with biennial or more complex epidemic patterns (e.g. measles), it is possible for pulse vaccination to drive the oscillations to an annual periodic attractor (figure $4 c$ ). This contrasts with the impact of routine vaccination against measles in England and Wales and the USA, where the increase in $T$ resulted in irregular, decorrelated epidemics (Bolker \& Grenfell 1996; Earn et al. 2000). The impact 

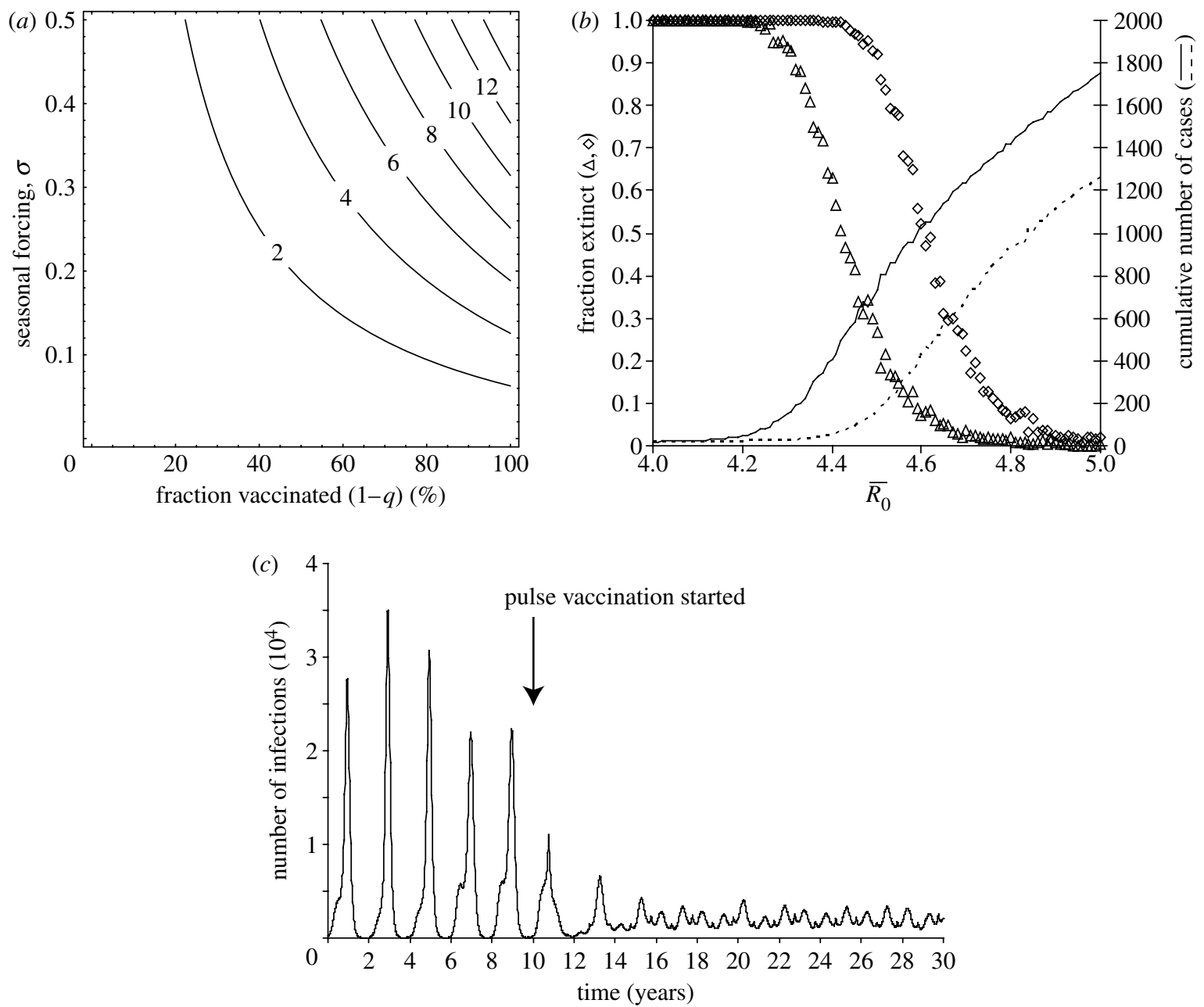

Figure 4. Pulse vaccination and seasonal infectious disease dynamics. (a) A contour plot showing the percentage reduction in $\bar{R}_{v}$ for annual pulse vaccination three months prior to peak transmission $(\delta=0.25)$, compared to six months $(\delta=0.5)$, for a seasonal infection with sinusoidal variation in transmission with strength $\sigma$ and vaccination coverage $(1-q)$. (b) Numerical simulations of a stochastic, sinusoidal seasonal SIR model showing the fraction of populations where the infection goes extinct and the cumulative cases of disease for an infection with robustly annual epidemics and pulse vaccination at six months prior to peak transmission $(\delta=0.5$; triangles, solid line) and at three months $(\delta=0.25$; diamonds, dashed line). Each point represents 100 simulations for 100 years post-vaccination, with model parameters $\sigma=0.6, \mu=0.16, N=10^{5}$ and $D=6$ weeks. Pulse vaccination is assumed to successfully immunize $40 \%$ of susceptible children $(q=0.6)$. (c) Prevalence of infection over time for a seasonal stochastic SIR model where the introduction of pulse vaccination at time 10 years results in a switch from biennial to annual epidemics. The model parameters are $\bar{R}_{0}=6, \sigma=0.3, N=10^{6}, \delta=0.25$ years and $D=2$ weeks.

of the introduction of an additional periodic attractor on persistence of infections with complex dynamics is unclear and likely to depend on population structure and patterns of contact. If prior to vaccination the complex epidemic patterns showed little temporal correlation between cities, then the synchronicity induced by pulse vaccination can increase the chance of global extinction (Earn et al. 1998). Conversely, for well-connected cities, patterns of incidence are likely to be strongly correlated (Grassly et al. 2005), in which case the unstable, longer period epidemics prior to the introduction of pulse vaccination would have been more likely to result in global extinction than the regular epidemics post-vaccination. The timing of immunization days must therefore be chosen with careful consideration of the nature of the seasonal forcing and the likely impact of vaccination on the dynamics of infection. It is conceivable that vaccination three months after peak transmission $(\delta=-0.25)$ to effectively increase the strength of seasonal forcing $\sigma$ could increase the likelihood of global eradication for infections prone to unstable dynamics.

\section{DISCUSSION}

Despite early interest in the causes of seasonal variation in infectious disease incidence (Ransome 1880; Hamer 1906; Soper 1929), they remain poorly understood more than a century later. There appear to be several reasons for this. First, the causes of seasonality are many, and their relative importance depends on the local context. For example, winter peaks in the incidence of measles in temperate regions are likely to be caused not only by school terms, but also by lower indoor humidity, which favours survival of the virus in the air (de Jong \& Winkler 1964). In the tropics, measles incidence peaks during the dry season and the association with school terms is not apparent. Instead, increased survival of the virus in the dryer air may be the key determinant of these dry season peaks.

Second, peaks in disease incidence can occur at significantly different times of year to peak transmission, depending on the characteristics of the infection (Dietz 1976). Infections with the same underlying cause of seasonal transmission may therefore show different 
patterns of incidence. Furthermore, in many cases, the interaction of the nonlinear dynamics of the infection with seasonal forcing of transmission results in more complex dynamics, including multi-annual cycles with variable periodicity (figure 3). These nonlinearities act to obscure correlation between climatic or behavioural factors and annual variation in disease incidence.

Finally, the dynamics of seasonal infections can be complicated by the interaction of the pathogens themselves, acting either to enhance the spread of one another through immune suppression (Lello et al. 2004) or antibody-dependent enhancement (Ferguson et al. 1999), or to inhibit one another through the removal of susceptible individuals by cross-immunity or mortality (Rohani et al. 2003; Lello et al. 2004). These interactions can lead to seasonal dynamics that would not be predicted by analysis of each infection in isolation (Rohani et al. 2003).

The problems these complications pose to the identification and quantifying of the causes of seasonality call for a comparative approach to analysing infectious disease data. Comparative analysis of longitudinal surveillance data for the same infection in different locations will help identify hypothesized causes of seasonality where these differ by location. For example, better surveillance of influenza in the tropics would allow a better understanding of the role of climate versus human contact patterns in driving seasonality.

Comparative analysis of different infections from the same location will also be useful, not only because infections can directly interact, but because different disease natural histories will lead to different temporal dynamics in response to the same seasonal forcing. Careful analysis of surveillance data can therefore allow fragile theories about mathematical complexity to be addressed and the causes of seasonal forcing more robustly identified.

Central to this work is the need to measure potential drivers of seasonality. In the case of climate, this may be relatively straightforward. However, patterns of human contact are difficult to describe mathematically, and much theoretical and empirical work remains to be done in this area.

Although the complex patterns of incidence that can result from seasonal forcing of transmission have been well studied, the implications for persistence remain poorly understood. This probably reflects the importance of spatial heterogeneities in transmission and migration, and the resulting patterns of local extinction, re-infection and global persistence, which are difficult to measure and analyse. Additional complications arise from the consideration of pathogen genetic variation. For example, the repeated 'bottlenecks' in the pathogen population caused by seasonality will restrict pathogen genetic (and antigenic) diversity, potentially with implications for disease persistence. Seasonal variation in transmission also has consequences for the size distribution of disease outbreaks, which can lead to overestimates of $R_{0}$ from surveillance data.

A better understanding of seasonal infectious disease outbreaks and persistence is likely to result in a better understanding of the optimal control strategies. However, it is also important to explicitly consider the interaction of control strategies themselves with seasonal dynamics.
In the case of pulse vaccination, we have shown the importance of considering the timing of immunization days. These considerations extend to programmes with periodic mass administration of antibiotics for seasonal infections, such as ocular Chlamydia trachomatis. In this case, however, the intervention results in abrupt periodic changes in the number of infected, rather than susceptible, individuals, and the optimal time for treatment may differ from that for vaccination (Lee et al. 2005).

Seasonality has moved from the centre ground of early infectious disease epidemiology to the periphery; a distraction often ignored or assumed of minor importance. This is surprising, given its prevalence of occurrence, significance for many key epidemiological parameters and intense interest from the non-specialist, as to why 'colds' occur in the winter when it is cold. Perhaps the greatest obstacle to progress has been the absence of sufficient comparative data with which to test hypotheses about causation and examine the dynamic consequences of seasonality. This is another reason for the improvement and expansion of routine surveillance of infectious diseases.

We would like to thank the Royal Society for funding, and Peter Aaby, Pete Dodd, Geoff Garnett, Déirdre Hollingsworth, Ben Lopman and James Truscott for helpful discussion. This paper benefits from suggestions made by anonymous referees during the peer-review process.

\section{REFERENCES}

Agur, Z., Cojocaru, L., Mazor, G., Anderson, R. M. \& Danon, Y. L. 1993 Pulse mass measles vaccination across age cohorts. Proc. Natl Acad. Sci. USA 90, 11 698-11 702.

Allen, J. C., Schaffer, W. M. \& Rosko, D. 1993 Chaos reduces species extinction by amplifying local population noise. Nature 364, 229-232. (doi:10.1038/364229a0)

Altizer, S., Dobson, A., Hosseini, P., Hudson, P., Pascual, M. \& Rohani, P. 2006 Seasonality and the dynamics of infectious diseases. F. Anim. Ecol. 9, 467-484.

Anderson, R. M. \& May, R. M. 1991 Infectious diseases of humans: dynamics and control. Oxford, UK: Oxford University Press.

Aron, J. L. \& Schwartz, I. B. 1984 Seasonality and period doubling bifurcations in an epidemic model. $\mathcal{F}$. Theor. Biol. 110, 665-679.

Bailey, N. T. J. 1975 The mathematical theory of infectious diseases and its applications, pp. 105-107, 2nd edn. London, UK: Griffin.

Bartlett, M. S. 1957 Measles periodicity and community size. F. R. Stat. Soc. A 120, 48-70.

Bauch, C. T. \& Earn, D. J. D. 2003 Transients and attractors in epidemics. Proc. R. Soc. B 270, 1573-1578. (doi:10. 1098/rspb.2003.2410)

Birmingham, M. E., Aylward, R. B., Cochi, S. L. \& Hull, H. F. 1997 National immunization days: state of the art. F. Infect. Dis. 175(Suppl. 1), S183-S188.

Blakebrough, I. S. 1979 Epidemiological and laboratory studies of meningococcal infections in Nigeria. Ph.D. thesis, University of Liverpool, UK.

Bolker, B. M. \& Grenfell, B. T. 1996 Impact of vaccination on the spatial correlation and persistence of measles dynamics. Proc. Natl Acad. Sci. USA 93, 12 648-12 653. (doi:10.1073/pnas.93.22.12648)

Chavasse, D. C., Shler, R. P., Murphy, O. A., Huttly, S. R. A., Cousens, S. N. \& Akhtar, T. 1999 Impact of fly control on 
childhood diarrhoea in Pakistan: community-randomised trial. Lancet 353, 22-25. (doi:10.1016/S0140-6736(98) 03366-2)

Chew, F. T., Doraisingham, S., Ling, A. E., Kumarasinghe, G. \& Lee, B. W. 1998 Seasonal trends of viral respiratory tract infections in the tropics. Epidemiol. Infect. 121, 121-128. (doi:10.1017/S0950268898008905)

Cook, S. M., Glass, R. I., Lebaron, C. W. \& Ho, M. S. 1990 Global seasonality of rotavirus infections. Bull. World Health Organ. 68, 171-177.

Davis, S., Begon, M., De Bruyn, L., Ageyev, V. S., Klassovskiy, N. L., Pole, S. B., Viljugrein, H., Stenseth, N. C. \& Leirs, H. 2004 Predictive thresholds for plague in Kazakhstan. Science 304, 736-738. (doi:10.1126/science. 1095854)

Deguen, S., Thomas, G. \& Chau, N. P. 2000 Estimation of the contact rate in a seasonal SEIR model: application to chickenpox incidence in France. Stat. Med. 19, 1207-1216. (doi:10.1002/(SICI)1097-0258(20000515) 19:9<1207::AID-SIM423>3.0.CO;2-L)

de Jong, J. G. \& Winkler, K. C. 1964 Survival of measles virus in air. Nature 201, 1054-1055.

Diekmann, O., Heesterbeek, J. A. P. \& Metz, J. A. J. 1990 On the definition and the computation of the basic reproduction ratio $R_{0}$ in models for infectious diseases in heterogeneous populations. F. Math. Biol. 28, 365-382. (doi:10.1007/BF00178324)

Dietz, K. 1976 The incidence of infectious diseases under the influence of seasonal fluctuations. In Lecture notes in biomathematics, vol. 11 (ed. J. Berger et al.), pp. 1-15. Berlin, Germany: Springer.

Dowell, S. F. 2001 Seasonal variation in host susceptibility and cycles of certain infectious diseases. Emerg. Infect. Dis. 7, 369-374.

Dushoff, J., Plotkin, J. B., Levin, S. A. \& Earn, D. J. D. 2004 Dynamical resonance can account for seasonality of influenza epidemics. Proc. Natl Acad. Sci. USA 101, 16 915-16 916. (doi:10.1073/pnas.0407293101)

Earn, D. J. D., Rohani, P. \& Grenfell, B. T. 1998 Persistence, chaos and synchrony in ecology and epidemiology. Proc. R. Soc. B 265, 7-10. (doi:10.1098/rspb.1998.0256)

Earn, D. J. D., Rohani, P., Bolker, B. M. \& Grenfell, B. T. 2000 A simple model for complex dynamical transitions in epidemics. Science 287, 667-670. (doi:10.1126/science. 287.5453.667)

Emerson, P. M., Bailey, R. L., Mahdi, O. S., Walraven, G. E. L. \& Lindsay, S. W. 2000 Transmission ecology of the fly Musca sorbens, a putative vector of trachoma. Trans. R. Soc. Trop. Med. Hyg. 94, 28-32. (doi:10.1016/S00359203(00)90427-9)

Farrington, C. P., Kanaan, M. N. \& Gay, N. J. 2003 Branching process models for surveillance of infectious diseases controlled by mass vaccination. Biostatistics 4, 279-295. (doi:10.1093/biostatistics/4.2.279)

Ferguson, N., Anderson, R. \& Gupta, S. 1999 The effect of antibody-dependent enhancement on the transmission dynamics and persistence of multiple-strain pathogens. Proc. Natl Acad. Sci. USA 96, 790-794. (doi:10.1073/ pnas.96.2.790)

Ferguson, N. M., Fraser, C., Donnelly, C. A., Ghani, A. C. \& Anderson, R. M. 2004 Public health risk from the avian H5N1 influenza epidemic. Science 304, 968-969. (doi:10. 1126/science.1096898)

Fine, P. \& Clarkson, J. 1982 Measles in England and Wales-I: an analysis of factors underlying seasonal patterns. Int. F. Epidemiol. 11, 5-14.

Finkenstadt, B. F. \& Grenfell, B. T. 2000 Time series modelling of childhood diseases: a dynamical systems approach. Appl. Stat. 49, 182-205.
Gay, N. J., De Serres, G., Farrington, C. P., Redd, S. B. \& Papania, M. J. 2004 Assessment of the status of measles elimination from reported outbreaks: United States, 1997-1999. F. Infect. Dis. 189(Suppl. 1), S36-S42. (doi:10.1086/377695)

Gomes, M. C., Gomes, J. J. \& Paulo, A. C. 1999 Diphtheria, pertussis, and measles in Portugal before and after mass vaccination: a time series analysis. Eur. F. Epidemiol. 15, 791-798. (doi:10.1023/A:1007615513441)

Grassly, N. C., Fraser, C. \& Garnett, G. P. 2005 Host immunity and synchronized epidemics of syphilis across the United States. Nature 433, 417-421. (doi:10.1038/ nature03072)

Greenwood, B. 1999 Meningococcal meningitis in Africa. Trans. R. Soc. Trop. Med. Hyg. 93, 341-353. (doi:10.1016/ S0035-9203(99)90106-2)

Grenfell, B. T., Bolker, B. M. \& Kleczkowski, A. 1995 Seasonality and extinction in chaotic metapopulations. Proc. R. Soc. B 259, 97-103.

Grenfell, B. T., Bjornstad, O. N. \& Kappey, J. 2001 Travelling waves and spatial hierarchies in measles epidemics. Nature 414, 716-723. (doi:10.1038/414716a)

Hamer, W. H. 1906 Epidemic disease in England: the evidence of variability and the persistence of type. Lancet 167, 733-739. (doi:10.1016/S0140-6736(01)80340-8)

Hay, S. I., Were, E. C., Renshaw, M., Noor, A. M., Ochola, S. A., Olusanmi, I., Alipui, N. \& Snow, R. W. 2003 Forecasting, warning, and detection of malaria epidemics: a case study. Lancet 361, 1705-1706. (doi:10.1016/S01406736(03)13366-1)

Heesterbeek, H. 2005 The law of mass-action in epidemiology: a historical perspective. In Ecological paradigms lost: routes of theory change (ed. K. Cuddington \& B. Beisner), pp. 81-105. Amsterdam, The Netherlands: Elsevier.

Heesterbeek, J. A. P. \& Roberts, M. G. 1995 Threshold quantities for helminth infections. F. Math. Biol. 33, 415-434. (doi:10.1007/BF00176380)

Hemmes, J. H., Winkler, K. C. \& Kool, S. M. 1960 Virus survival as a seasonal factor in influenza and poliomyelitis. Nature 188, 430-431.

Hethcote, H. W. \& Yorke, J. A. 1984 Gonorrhea transmission dynamics and control. Lecture notes in biomathematics, vol. 56, p. 105. Berlin, Germany: Springer.

Heymann, D. L. \& Aylward, R. B. 2004 Eradicating polio. N. Engl. F. Med. 351, 1275-1277. (doi:10.1056/NEJMp 048204)

Jagers, P. \& Nerman, O. 1985 Branching processes in periodically varying environments. Ann. Prob. 13, 254-268.

Jansen, V. A. A., Stollenwerk, N., Jensen, H. J., Ramsay, M. E., Edmunds, W. J. \& Rhodes, C. J. 2003 Measles outbreaks in a population with declining vaccine uptake. Science 301, 804. (doi:10.1126/science.1086726)

John, T. J., Pandian, R., Gadomski, A., Steinhoff, M., John, M. \& Ray, M. 1983 Control of poliomyelitis by pulse immunisation in Vellore, India. Br. Med. F. (Clin. Res. Ed.) 286, 31-32.

Keeling, M. J., Rohani, P. \& Grenfell, B. T. 2001 Seasonally forced disease dynamics explored as switching between attractors. Physica D 148, 317-335.

Lee, D. C., Chidambaram, J. D., Porco, T. C. \& Lietman, T. M. 2005 Seasonal effects in the elimination of trachoma. Am. F. Trop. Med. Hyg. 72, 468-470.

Lello, J., Boag, B., Fenton, A., Stevenson, I. R. \& Hudson, P. J. 2004 Competition and mutualism among the gut helminths of a mammalian host. Nature 428, 840-844. (doi:10.1038/nature02490)

London, W. P. \& Yorke, J. A. 1973 Recurrent outbreaks of measles, chickenpox and mumps. I. Seasonal variation in contact rates. Am. F. Epidemiol. 98, 453-468. 
Loosli, C. G., Lemon, H. M., Robertson, O. H. \& Appel, E. 1943 Experimental airborne influenza infection. I. Influence of humidity on survival of virus in air. Proc. Soc. Exp. Biol. Med. 53, 205-206.

Lord, C. C., Woolhouse, M. E. J., Heesterbeek, J. A. P. \& Mellor, P. S. 1996 Vector-borne diseases and the basic reproduction number: a case study of African horse sickness. Med. Vet. Entomol. 10, 19-28.

Ma, J. L. \& Ma, Z. 2006 Epidemic threshold conditions for seasonally forced SEIR models. Math. Biosci. Eng. 3, 161-172.

Mabaso, M. L. H., Craig, M., Vounatsou, P. \& Smith, T. 2005 Towards empirical description of malaria seasonality in southern Africa: the example of Zimbabwe. Trop. Med. Int. Health 10, 909-918. (doi:10.1111/j.1365-3156.2005. 01462.x)

Mounts, A. W., Ando, T., Koopmans, M., Bresee, J. S., Noel, J. \& Glass, R. I. 2000 Cold weather seasonality of gastroenteritis associated with Norwalk-like viruses. F. Infect. Dis. 181(Suppl. 2), S284-S287. (doi:10.1086/ 315586)

Nåsell, I. 2002 Stochastic models of some endemic infections. Math. Biosci. 179, 1-19. (doi:10.1016/S00255564(02)00098-6)

Nelson, R. J. \& Demas, G. E. 1996 Seasonal changes in immune function. Q. Rev. Biol. 71, 511-548. (doi:10. 1086/419555)

Nokes, D. J. \& Swinton, J. 1995 The control of childhood viral infections by pulse vaccination. IMA F. Math. Appl. Med. Biol. 12, 29-53.

Nokes, D. J. \& Swinton, J. 1997 Vaccination in pulses: a strategy for global eradication of measles and polio? Trends Microbiol. 5, 14-19. (doi:10.1016/S0966-842X(97)81769-6)

Pascual, M., Rodo, X., Ellner, S. P., Colwell, R. \& Bouma, M. J. 2000 Cholera dynamics and El Niño-Southern Oscillation. Science 289, 1766-1769.

Pascual, M., Bouma, M. J. \& Dobson, A. P. 2002 Cholera and climate: revisiting the quantitative evidence. Microbes Infect. 4, 237-245. (doi:10.1016/S1286-4579(01)01533-7)

Ramsay, M. E., Jin, L., White, J., Litton, P., Cohen, B. \& Brown, D. 2003 The elimination of indigenous measles transmission in England and Wales. F. Infect. Dis. 187(Suppl. 1), S198-S207. (doi:10.1086/368024)
Randolph, S. E. 2004 Tick ecology: processes and patterns behind the epidemiological risk posed by ixodid ticks as vectors. Parasitology 129, S37-S65. (doi:10.1017/ S0031182004004925)

Ransome, A. 1880 On epidemic cycles. Proc. Manchester Lit. Phil. Soc. 19, 75-96.

Rohani, P., Keeling, M. J. \& Grenfell, B. T. 2002 The interplay between determinism and stochasticity in childhood diseases. Am. Nat. 159, 469-481. (doi:10.1086/ 339467)

Rohani, P., Green, C. J., Mantilla-Beniers, N. B. \& Grenfell, B. T. 2003 Ecological interference between fatal diseases. Nature 422, 885-888. (doi:10.1038/nature01542)

Schwartz, I. B. 1985 Multiple stable recurrent outbreaks and predictability in seasonally forced nonlinear epidemic models. f. Math. Biol. 21, 347-361. (doi:10.1007/ BF00276232)

Schwartz, I. B. 1992 Small amplitude, long period outbreaks in seasonally driven epidemics. F. Math. Biol. 30, 473-491. (doi:10.1007/BF00160532)

Soper, H. E. 1929 The interpretation of periodicity in disease prevalence. F. R. Stat. Soc. 92, 34-73.

Sturrock, R. F., Diaw, O. T., Talla, I., Niang, M., Piau, J. P. \& Capron, A. 2001 Seasonality in the transmission of schistosomiasis and in populations of its snail intermediate hosts in and around a sugar irrigation scheme at Richard Toll, Senegal. Parasitology 123, S77-S89. (doi:10.1017/ S0031182001008125)

Sultan, B., Labadi, K., Guégan, J.-F. \& Janicot, S. 2005 Climate drives the meningitis epidemics onset in West Africa. PLoS Med. 2, e6. (doi:10.1371/journal.pmed. 0020006)

Trapman, P., Meester, R. \& Heesterbeek, H. 2004 A branching model for the spread of infectious animal diseases in varying environments. F. Math. Biol. 49, 553-576. (doi:10.1007/s00285-004-0267-5)

Williams, B. G. \& Dye, C. 1997 Infectious disease persistence when transmission varies seasonally. Math. Biosci. 145, 77-88. (doi:10.1016/S0025-5564(97)00039-4)

Yorke, J. A., Nathanson, N., Pianigiani, G. \& Martin, J. 1979 Seasonality and the requirements for perpetuation and eradication of viruses in populations. Am. F. Epidemiol. 109, 103-123. 